\title{
Magnetic Moments of Baryons containing all heavy quarks in Quark-Diquark Model
}

\author{
Kaushal Thakkar ${ }^{1,}$, Ajay Majethiya ${ }^{2}$ and P. C. Vinodkumar ${ }^{3}$ \\ 1 Department of Applied Sciences \& Humanities, GIDC Degree Engineering College, Abrama, Navsari-396406, India \\ 2 V S Patel College of Arts and Science, Bilimora-396321, India \\ 3 Department of Physics, Sardar Patel University, Vallabh Vidyanagar-388 120, India \\ ${ }^{*}$ Corresponding Author: kaushal2physics@gmail.com
}

Received: date / Revised version: date

\begin{abstract}
The triply heavy flavour baryons are studied using the Quark-diquark description of the threebody system. The confinement potential for present study of triply heavy flavour baryons is assumed as coulomb plus power potential with power index $\nu$. We have solved Schrodinger equation numerically to calculate the masses of triply heavy flavour baryons. The masses and magnetic moments of triply heavy flavour baryons are computed for different power indices, $\nu$, starting from 0.4 to 1.0. The predicted masses and magnetic moments are in good agreement with other theoretical predictions.
\end{abstract}

PACS. 12.39.-x Phenomenological quark models - 12.39.Jh Nonrelativistic quark model - 14.20.-c Baryons

\section{Introduction}

The field of heavy baryon spectroscopy is experiencing a rapid advancement in recent times. Numerous experimental data on heavy flavour baryons are reported. Particle Data Group (PDG 2014) has listed ground states of heavy baryons with single heavy quark [1]. Though theoretical data on the properties of heavy flavour baryons are available in literature, the ground state masses of doubly and triply heavy flavour baryons have not been measured experimentally. The SELEX Collaboration have claimed the observation of a $\Xi_{C C}^{+}$double charm baryon through the decay channel from all possible doubly heavy flavour baryon states 2, 3,4. Thus the theoretical predictions of the doubly and triply heavy baryon mass spectrum have become a subject of renewed interest and are relevant due to the recent experimental efforts at CMS, SELEX, $\mathrm{LHCb}$ etc. These experimental groups have been successful in discovering heavy baryonic states along with other heavy flavour mesonic states and it is expected that more heavy flavour baryon states will be detected at J-PARC, PANDA, LHCb, and Belle II in their future efforts.

As in the case of $\Delta^{++}$(uuu), $\Delta^{-}$(ddd) and $\Omega^{-}$(sss) baryons in the light flavour sector, QCD predicts similar states made up of charm quarks, $\Omega_{c c c}^{++}$(ccc) and bottom quarks, $\Omega_{b b b}^{-}$(bbb) in the heavy flavour sector. Though such a state yet to be observed experimentally. After the observation of the doubly charmed baryon by the SELEX group, it is expected that the triply heavy flavour baryonic state may be in the offing very soon. Though considerable amount of data on the properties of the singly-heavy baryons are available in literature $[5,6,7,8,9,10,11,12,13$, only sparse attention has been paid to the spectroscopy of double and triple-heavy flavour baryons, perhaps due to the lack of experimental incentives. Theoretically, baryons are not only the interesting systems to study the quark dynamics and their properties but are also interesting from the point of view of simple systems to study three body problems. Out of many approaches and methods such as the QCD sum rules, QCD bag model, the effective field theory, lattice QCD, hyper central model, potential model etc., available for the three body systems $14,15,16,17,18$, 19, 20, 21, we employ here the Quark-diquark approach to study the triply heavy flavour baryons. The magnetic moments of heavy flavour baryons are computed based on the non-relativistic quark model using the spin-flavour wave functions of the constituting quarks. The binding energy effects are considered by defining an effective mass of the bound quarks within the baryon for computing the magnetic moments. In the present study, we compute the effective masses of the constituent quarks within the baryon with different combinations of heavy flavour quarks. We repeat our computations by varying the power index $\nu$ of confinement potential from 0.4 to 1.0 to have an idea about the most suitable form of inter quark potential that yields the static properties of the triple heavy baryons.

The study presented in this paper is organized as follows: In section 2 the Quark-diquark model and a brief introduction of Coulomb plus power potential employed for the present study are described. The details of magnetic moments and its dependence of confinement through effective quark mass are presented in section 3 . We anal- 
yse and discuss our results and make our own conclusions in section 4.

\section{Theoretical Framework: Quark-diquark model}

For the description of the 3-body system like the baryons, we have employed the quark-diquark model with interquark potential of the coulomb plus power potential form to study the masses and magnetic moments of baryons containing three heavy flavour quarks. Following Gell-Mann's suggestion of the possibility of quark-diquark structure for baryons [22], various authors have introduced effective degrees of freedom of diquarks in order to describe baryons as composed of a constituent diquark and quark [23,24, 25, 26. The presence of a coherent diquark structure within baryons helps us to treat the problem of three-body interaction to that of two two-body interactions.

In this case, Hamiltonian of the baryon is expressed in terms of a diquark Hamiltonian $\left(H_{j k}\right)$ plus quark-diquark Hamiltonian $\left(H_{i, j k}\right)$ as 27,28.

$$
H=H_{j k}+H_{i, j k}
$$

The internal motion of the $\operatorname{diquark}(j k)$ is described by

$$
H_{j k}=\frac{p^{2}}{2 m_{j k}}+V_{j k}\left(r_{j k}\right)
$$

and the Hamiltonian of the relative motion of the $\operatorname{diquark}(d)$ quark $(i)$ system is described by

$$
H_{i, d}=H_{i, j k}=\frac{q^{2}}{2 m_{i, j k}}+V_{i, j k}\left(r_{i d}\right)
$$

where, $\left(p, r_{j k}\right)$ represent the relative momenta and coordinate of the quarks within the diquark system and $\left(q, r_{i d}\right)$ is the relative momenta and coordinate of the quark-diquark system. The reduced mass of the two body systems appeared in Eqn.(2) and Eqn.(3) respectively are defined as

$$
m_{j k}=\frac{m_{j} m_{k}}{m_{j}+m_{k}}, m_{i, j k}=\frac{m_{i}\left(m_{j}+m_{k}\right)}{m_{i}+m_{j}+m_{k}}
$$

For the present study, we have assumed colour coulomb plus power potential for the interquark potential of Eqn.(2) as well as for the quark-diquark interaction of Eqn.(3). Accordingly, the diquark potential can be written as,

$$
V_{j k}=-\frac{2}{3} \alpha_{s} \frac{1}{r_{j k}}+b r_{j k}^{\nu}
$$

and the quark-diquark potential as

$$
V_{i, j k}=-\frac{4}{3} \alpha_{s} \frac{1}{r_{i d}}+b r_{i d}^{\nu}
$$

where, $r_{i d}$ is the quark-diquark separation distance, $\nu$ is the exponent corresponding to the confining part of the
Table 1. Model parameters for $\Omega_{c c c}$ systems in quark-diquark model.

\begin{tabular}{cccc}
\hline \hline$\alpha_{s}$ & $\nu$ & $b$ & $m_{c}$ \\
\hline & 0.4 & 0.1975 & 1.360 \\
& 0.6 & 0.1010 & 1.435 \\
0.20 & 0.8 & 0.054 & 1.480 \\
& 1.0 & 0.033 & 1.500 \\
\hline \hline
\end{tabular}

Table 2. Model parameters for $\Omega_{c c b}$ and $\Omega_{b b c}$ systems in quark-diquark model.

\begin{tabular}{ccccc}
\hline \hline$\alpha_{s}$ & $\nu$ & $b$ & $m_{c}$ & $M_{b}$ \\
\hline & 0.4 & 0.1875 & 1.360 & 4.720 \\
& 0.6 & 0.0955 & 1.435 & 4.780 \\
0.17 & 0.8 & 0.0495 & 1.480 & 4.815 \\
& 1.0 & 0.0282 & 1.500 & 4.840 \\
\hline \hline
\end{tabular}

potential and $b$ is the strength of the potential, which is assumed to be same for the di-quark interaction as well as between the quark-diquark interaction. The Schrodinger equation corresponds to the Hamiltonian given in Eqn.(1) is numerically solved using the Runge-Kutta method. The degeneracy of the states are removed by introducing the spin dependent interaction potential given by 29 ]

$$
V_{S D}^{(d)}\left(r_{j k}\right)=\frac{2}{3} \alpha_{s} \frac{1}{3 m_{j} m_{k}} \mathbf{S}_{\mathbf{j}} \cdot \mathbf{S}_{\mathbf{k}}\left[4 \pi \delta\left(r_{j k}\right)\right]
$$

among the diquark states, and

$$
V_{S D}^{(i-d)}(r)=\frac{4}{3} \alpha_{s} \frac{1}{3 m_{i} 2 m_{j k}}\left(\mathbf{S}_{\mathbf{d}}+\mathbf{L}_{\mathbf{d}}\right) \cdot \mathbf{S}_{\mathbf{q}}\left[4 \pi \delta\left(r_{i d}\right)\right]
$$

among the quark-diquark $(i d)$ system.

The model parameters are fixed for the potential index $\nu=0.4$ to 1.0 to get the spin average mass of the system $\Omega_{c c c}^{++}$(4746), $\Omega_{c c b}^{+}(8021), \Omega_{b b c}^{+}(11283), \Omega_{b b b}^{-}(14370)$. All other properties are predicted without changing any of these parameters. The model parameters of triple heavy flavour baryons are shown in Table 1 2 and 3. Our results for $J^{P}=\frac{1}{2}^{+}$and $\frac{3}{2}^{+}$are also compared with other theoretical model predictions. It is interesting to note that our predictions are in good agreement with other theoretical model predictions. The mass variations of the heavy baryons with respect to $\nu$ from 0.4 to 1.0 are found to be around $100 \mathrm{MeV}$ only.

\section{Magnetic moments of QQQ baryons}

According to the idea of constituent quark mass [30, the hadron mass $\mathrm{M}$ is given by the sum of the masses of its constituent quarks. Accordingly, the kinetic and binding energies must be incorporated in the constituent quark mass. 
Table 3. Model parameters for $\Omega_{b b b}$ system in quark-diquark model.

\begin{tabular}{lccc}
\hline \hline$\alpha_{s}$ & $\nu$ & $b$ & $m_{b}$ \\
\hline 0.17 & 0.4 & 0.097 & 4.720 \\
& 0.6 & 0.015 & 4.780 \\
\hline \hline
\end{tabular}

Table 4. Triply heavy baryon masses (in MeV) in quarkdiquark model

\begin{tabular}{crllll}
\hline \hline Baryon & Model & $\mathbf{J}^{P}=\frac{1}{2}^{+}$ & Others & $\mathbf{J}^{P}=\frac{3}{2}^{+}$ & O \\
\hline$\Omega_{c c c}^{++}$ & $\nu=0.4$ & - & - & 4760 & 4965 \\
& 0.6 & - & - & 4760 & 4760 \\
& 0.8 & - & - & 4760 & 480 \\
& 1.0 & - & - & 4760 & 4790 \\
& & - & - & & 4773
\end{tabular}

$\begin{array}{lrlllll}\Omega_{c c b}^{+} & \nu= & 0.4 & 7999 & 8245[33 & 8032 \\ & 0.6 & 8002 & 8018[3] & 8031 \\ & 0.8 & 8004 & - & 8028 \\ & 1.0 & 8005 & 7984 & 37] & 8027\end{array}$

$\begin{array}{lrllll}\Omega_{b b c}^{0} & \nu= & 0.4 & 11274 & 11535[\underline{33} & 11287 \\ & 0.6 & 11275 & 11280[\underline{34} & 11286 \\ & 0.8 & 11276 & & & 11285 \\ & 1.0 & 11277 & 11139 & {[37} & 11284\end{array}$

$\begin{array}{rrrrr}\Omega_{b b b}^{-} & \nu=0.4 & - & - & 14370 \\ & 0.6 & - & - & 14370 \\ 0.8 & - & - & 14370 \\ 1.0 & - & - & 14370 \\ & & - & - & \end{array}$

Table 5. Spin-flavour wave functions and magnetic moments of triple heavy flavour baryons.

\begin{tabular}{ll}
\hline \hline Baryon & Spin-flavour wave function \\
\hline$\Omega_{c c c}^{*++}$ & $c_{+} c_{+} c_{+}$ \\
$\Omega_{c c b}^{+}$ & $\frac{\sqrt{2}}{6}\left(2 b_{-} c_{+} c_{+}-c_{-} b_{+} c_{+}-b_{+} c_{-} c_{+}+2 c_{+} b\right.$ \\
& $-c_{-} c_{+} b_{+}-c_{+} b_{+} c_{-}-b_{+} c_{+} c_{-}+2 c_{+} c$ \\
$\Omega_{c c b}^{*+}$ & $\frac{1}{\sqrt{3}}\left(c_{+} c_{+} b_{+}+c_{+} b_{+} c_{+}+b_{+} c_{+} c_{+}\right)$ \\
$\Omega_{b b c}^{0}$ & $\frac{\sqrt{2}}{6}\left(2 c_{-} b_{+} b_{+}-b_{-} c_{+} b_{+}-c_{+} b_{-} b_{+}+2 b_{+} c\right.$ \\
& $-b_{-} b_{+} c_{+}-b_{+} c_{+} b_{-}-c_{+} b_{+} b_{-}+2 b_{+} b$ \\
$\Omega_{b b c}^{* 0}$ & $\frac{1}{\sqrt{3}}\left(b_{+} b_{+} c_{+}+b_{+} c_{+} b_{+}+c_{+} b_{+} b_{+}\right)$ \\
\hline \hline$\underline{\mathrm{rs} \Omega_{b b b}^{*-}}$ & $b_{+} b_{+} b_{+}$ \\
\hline \hline
\end{tabular}

$4965\left[3 \beta^{k}\right.$ indicates $J^{P}=\frac{3}{2}^{+}$state.)

480334

479035

4773 cordingly, the effective mass of the $c$ and $b$ quarks will 4777 B] different when it is in the baryonic states of ccb com-

binations or bbc combinations as $\langle H\rangle_{c c b} \neq\langle H\rangle_{b b c}$. The 8265 3effective masses of the constituting quarks in the case of 7980 Hige typical baryonic states are listed in the Table 6. 802534

820035 The magnetic moment of baryons are obtained in terms 800537 the spin, charge and effective mass of the bound quarks 11554 as 73 , 31

$11554\left[\begin{array}{ll}33 \\ 11190[19\end{array}\right.$

1128734

11480 are

11163 37.

$$
\begin{gathered}
\mu_{B}=\sum_{i}\left\langle\phi_{s f}\left|\mu_{i z}\right| \phi_{s f}\right\rangle \\
\mu_{i z}=\frac{e_{i} \sigma_{i z}}{2 m_{i}^{\text {eff }}}
\end{gathered}
$$

${ }_{14834} \mathrm{H3}$ e, $e_{i}$ and $\sigma_{i z}$ represents the charge and the spin pro14370 j 1456 in $145690^{\mathrm{S}} \mathrm{34}$ - flavour wave function and effective masses, we com$14276 \mathrm{P} 35$ the magnetic moments of the triple heavy flavour 14276 the
We define an effective mass to the constituting quarks within the baryons that takes care of the bound state effects including its internal motions and interactions among quarks as 31,32]

$$
m_{i}^{e f f}=m_{i}\left(1+\frac{\langle H\rangle}{\sum_{i} m_{i}}\right)
$$

For the computation of the magnetic moments, we consider the mass of bound quarks inside the baryons as its effective mass taking in to account of its binding interactions with other two quarks described by the Hamiltonian given in Eqn.(11). The effective mass for each of the constituting quark $m_{i}^{e f f}$ as defined in Eqn.(9), where, $\langle H\rangle=E+\left\langle V_{\text {spin }}\right\rangle$ are computed such that the corresponding mass of the baryon with spin angular momentum, $\mathrm{J}$ is given by

$$
M_{B}^{J}=\sum_{i} m_{i}+\langle H\rangle_{J}=\sum_{i} m_{i}^{e f f}
$$

The spin flavour wave functions and the magnetic moments in terms of their constituent quark magnetic moments corresponds to the various combinations of b, c quarks composition are listed in Table 5. Using the ground state masses predicted for the triple heavy flavour (QQQ, $\mathrm{Q} \in \mathrm{c}, \mathrm{b}$ ) baryons based on Quark-diquark approaches as discussed in section 2, we compute the effective masses of the constituting heavy quarks. The predicted magnetic moments of all the spin $J^{P}=\frac{1}{2}^{+}$and spin $J^{P}=\frac{3}{2}^{+}$triple heavy baryons based on quark-diquark model are listed in Table (7).

\section{Results and Discussions}

The masses and magnetic moments of triply heavy baryons in the Quark-diquark approach with coulomb plus power potential have been studied. It is interesting to note that our predictions of the mass of QQQ $(\mathrm{Q} \in \mathrm{c}, \mathrm{b})$ are in good agreement with existing predictions based on other theoretical models. 
Table 6. Effective quark mass (in $\mathrm{MeV}$ ) of triple heavy flavour baryons.

\begin{tabular}{ccc}
\hline \hline Baryon & $m_{c}^{\text {eff }}$ & $m_{b}^{\text {eff }}$ \\
\hline$\Omega_{c c c}^{++}$ & 1586 & - \\
\hline$\Omega_{c c b}^{+}$ & 1531 & 4936 \\
\hline$\Omega_{c c b}^{*+}$ & 1535 & 4951 \\
\hline$\Omega_{b b c}$ & 1512 & 4881 \\
\hline$\Omega_{b b c}^{*+}$ & 1514 & 4890 \\
\hline$\Omega_{b b b}^{-}$ & - & 4790 \\
\hline \hline
\end{tabular}

Table 7. Magnetic moments of triply heavy baryons in quarkdiquark model in units of Nuclear magneton $\mu_{N}$

\begin{tabular}{ccccc}
\hline \hline & \multicolumn{4}{c}{ Potential index $\nu$} \\
\cline { 2 - 5 } Baryon & 0.4 & 0.6 & 0.8 & 1.0 \\
\hline$\Omega_{c c c}^{++}$ & 1.182 & 1.182 & 1.182 & 1.18 \\
\hline$\Omega_{c c b}^{+}$ & 0.601 & 0.576 & 0.568 & 0.565 \\
\hline$\Omega_{c c b}^{*+}$ & 0.807 & 0.768 & 0.755 & 0.75 \\
\hline$\Omega_{b b c}^{+}$ & -0.235 & -0.227 & -0.224 & -0.223 \\
\hline$\Omega_{b b c}^{*+}$ & 0.325 & 0.297 & 0.288 & 0.28 \\
\hline$\Omega_{b b b}^{-}$ & -0.196 & -0.196 & -0.196 & -0.196 \\
\hline \hline${ }^{*}$ indicates $J^{P}=\frac{3}{2}^{+}$state.) & & & &
\end{tabular}

The predictions of the magnetic moment of triply heavy flavour baryons studied here are with no additional free parameters. Our results for magnetic moments of triply heavy flavour baryons are listed in Table (7) and are compared with other model predictions. The inter-quark interactions within the baryons are considered in the calculation of magnetic moments through the definition of effective mass of the constituent quarks within the baryon (Eqn. 9).

Our predicted results for masses of $\Omega_{c c c}^{+}$and $\Omega_{b b b}^{-}$are in good agreement with the masses predicted by [19, while our predictions for $\Omega_{c c b}^{+}$and $\Omega_{b b c}^{0}$ are in good agreement with masses predicted by 34. Our results for the magnetic moments of $\Omega_{c c c}^{+}$and $\Omega_{b b b}^{-}$are very close with NRQM predictions made by 39 . Our results on the magnetic moments of $\Omega_{c c b}^{+}$and $\Omega_{b b c}^{+}$are in agreement with the magnetic moments predicted by 38 .

It is interesting to note that the masses and magnetic moments predicted in our model do not vary appreciably with different choices of $\nu$ running from 0.4 to 1.0 as seen from Table (4) and Table (7). We look forward to see experimental data on these properties of triply heavy flavour baryons from future experimental facilities.

\section{References}

1. K.A. Olive et al., Chin. Phys. C 38, 090001 (2014).

2. M. Mattson et al., SELEX Collaboration, Phys. Rev. Lett. 89, 112001 (2002).
3. A. Ocherashvili et al., SELEX Collaboration, Phys. Lett. B 628, 18 (2005).

4. J. Eigelfried et al., SELEX Collaboration, Nucl. Phys. A 752, 121 (2005).

5. Zalak Shah, Kaushal Thakkar, Ajay Kumar Rai and P. C. Vinodkumar, arXiv:1602.06384 2 [hep-ph] (2016).

6. Kaushal Thakkar and P. C. Vinodkumar, proceedings of Science Hadron 2013, 055 (2013).

7. Ajay Majethiya, Bhavin Patel, P. C. Vinodkumar Eur. Phys. J. A 38:307-315 (2008).

8. D. Ebert, R. N. Faustov and V. O. Galkin, Phys. Rev. D 84, 014025 (2011).

9. H.-X. Chen, W. Chen, Q. Mao, A. Hosaka, et al., Phys. Rev. D 91, 054034 (2015).

10. T. Yoshida, E. Hiyama, A. Hosaka, M. Oka, and K. Sadato, Phys. Rev. D 92, 114029 (2015).

11. R. Bijker, F. Iachello and E. Santopinto, J Phys. A 31, 9Q1QN(1038) NRQM 38] NRQM 39.

9Q1QN(383) NRQM 38] NRQM 39].

12. L. Ya. Glozman-and D. O. Rige8, Phys. Rep. C 268, 263

751 U. Loring, K. Kretzschmar, B. Ch. Metsch, and H. R.

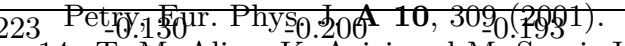

14. T. M. Aliev, K. Azizi and M. Savci, Journal of Physics G $41(6), 065003(2014)$.

Nilmani Mathur and Michael Peardon, Phys. Rev. D 90, 074504 (2014).

16. T. M. Aliev, K. Azizi and M. Savci, Journal of High Energy Physics 2013(4), 1-10 (2013).

17. Stefan Meinel, Phys. Rev. D 85, 114510 (2012).

18. Felipe J. Llanes-Estrada, Olga I. Pavlova and Richard Williams, European Physical Journal C 72(6), 1-21 (2012). 19. Yu Jia, Journal of High Energy Physics 10, 073 (2006).

20. J. Vijande, A. Valcarce and H. Garcilazo, Phys. Rev. D 91, 054011 (2015).

21. Bhavin Patel, Ajay Majethiya and P. C. Vinodkumar, Pramana Journal of Physics 72, 679-688 (2009).

22. M. Gell-Mann, Phys. Lett. 8, 214 (1964).

23. M. Ida et al., Progr. Theor. Phys. 8, 846 (1966).

24. D. B. Lichtenberg et al., Phys. Rev. 155, 1601 (1967).

25. M. Anselmino, E. Predazzi, S. Ekelin, S. Frederksson and

D. B. Lichtenberg, Rev. Mod. Phys. 65, 1199 (1993).

26. R. L. Jaffe et al., Phys. Rev. Lett. 91, 232003 (2003).

27. W. S. Carvalho et al., arXiv:hep-ph 9404298v1,(1994).

28. A. Majethiya, K. Thakkar and P. C. Vinodkumar, Chinese Journal of Physics 54, 495502 (2016).

29. S. S. Gershtein et al., Heavy Ion Physics 9 , 133-144 (1999); Phys. Atom. Nucl., 63; 274-286,(2000).

30. Ya. B. Zeldovich et al., Sov. J. Nucl. Phys. 4, 283 (1967).

31. B. Patel et al., J. Phy. G 35, 065001 (2008).

32. K. Thakkar, B. Patel, A. Majethiya and P. C. Vinodkumar, Pramana J. Phys. 77, 1053 (2011).

33. W. Roberts and Muslema Pervin, Int. Jn. Mod. Phys. A 23, 2817 (2008).

34. A. P. Martynenko, Phys. Lett. B 663, 317 (2008).

35. J. D. Bjorken, FERMILAB-Conf-85/69 (1985).

36. S. Migura, D. Merten, B. Metsch and H. -R. Petry, Eur. Phys. J. A 28, 41 (2006).

37. A. Bernotas and V.Šimonis, Lith. J. Phys. 49, 1928 (2009).

38. A. Faessler et al., Phys. Rev. D 73 094013(2006).

39. B. Silvestre-Brac, Prog. Part. Nucl. Phys.36, 263 (1996). 